\title{
Amplitude Modulation of Cepheid Radial Velocity Curves as a Systematic Source of Uncertainty for Baade-Wesselink Distances
}

\author{
Richard I. Anderson \\ Observatoire de Genève, Université de Genève, 51 Ch. des Maillettes, 1290 Sauverny, \\ Switzerland \\ email: richard.anderson@unige.ch
}

\begin{abstract}
I report on the recent discovery of modulation in the radial velocity curves in four classical Cepheids. This discovery may enable significant improvements in the accuracy of BaadeWesselink distances by revealing a not previously considered systematic source of uncertainty.
\end{abstract}

Keywords. techniques: radial velocities, stars: individual $\ell$ Carinae, stars: oscillations, supergiants, Cepheids, distance scale

\section{Introduction}

Cepheids are excellent standard candles thanks to a relation between the logarithm of the period and their intrinsic brightness (Leavitt \& Pickering 1912). This periodluminosity relation (PLR) finds application on Galactic and extragalactic scales and is crucial to precisely measure the expansion rate of the universe (Riess et al. 2011).

Baade-Wesselink (BW) type methods determine geometric distances by comparing angular and linear radius variations due to pulsation. They have been applied both to Cepheids in the Galaxy and the Magellanic Clouds (see Gieren et al. 2013, and references therein). Hence, different metallicities are probed using a homogeneous method, which is of great value regarding a possible metallicity dependence of the PLR. However, the accuracy of BW-type distances has suffered from certain difficulties usually ascribed to the projection (p-)factor (e.g. Nardetto et al. 2009), which is used to translate measured radial velocity into pulsation velocity.

\section{Radial Velocity Amplitude Modulations}

Using high-precision radial velocities $(\mathrm{RVs})$ obtained with the Coralie spectrograph mounted to the Swiss Euler telescope at La Silla, Anderson (2014) was recently able to show that Cepheid radial velocities are subject to modulation. This was demonstrated for two short-period, as well as two long-period Cepheids. Figure 1 exemplifies this phenomenon for the long-period $\ell$ Carinae.

\section{A Systematic Uncertainty for BW distances}

BW-type methods determine distance from the ratio of linear $(\Delta R)$ and angular $(\Delta \theta)$ radius variations due to pulsation. $\Delta R$ is computed as the integral of the pulsational velocity curve and therefore depends on the epoch of observation, if modulation is present. In consequence, combining measurements of $\Delta \theta$ from one epoch with $\Delta R$ from another epoch can lead to systematic errors in the derived distance, reaching up to $5-15 \%$ 


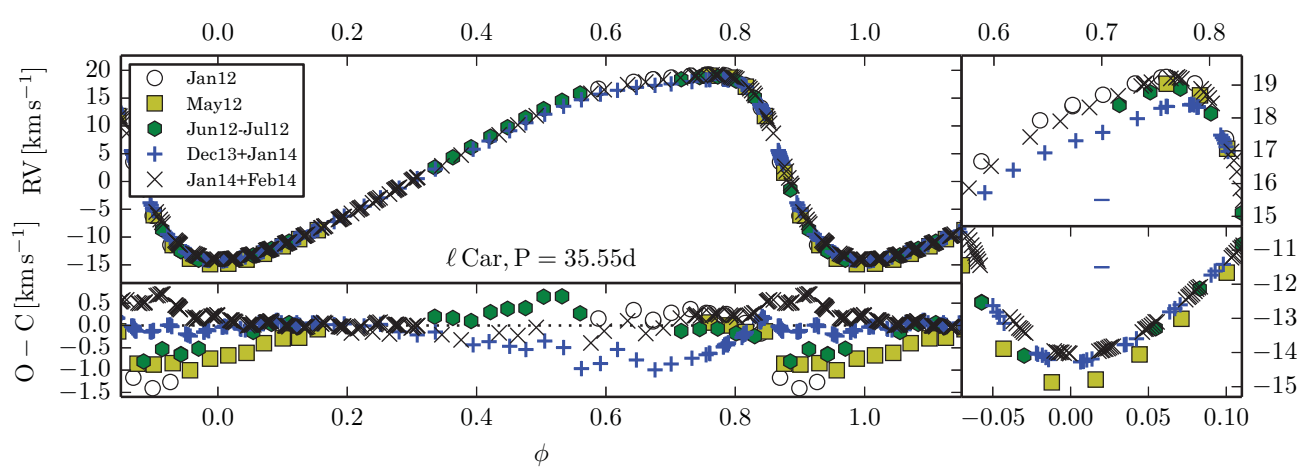

Figure 1. The modulated radial velocity curve of $\ell$ Carinae. Figure from Anderson (2014).

(Anderson 2014). It is thus crucial to measure angular and linear radius variations contemporaneously (equivalent pulsation cycles). While the impact of modulation on the distance estimate may average out over very long timescales (if the mean RV curve is stable), it appears likely that modulation explains a good portion of what has thus far limited the accuracy of BW distances.

\section{The way forward}

There are several possible explanations for the origin of RV modulations. From the fact that modulations in short-period Cepheids appear steady over longer timescales (years), while long-period Cepheids exhibit seemingly stochastic (cycle-to-cycle) modulation, it appears likely that different effects are responsible for the observed phenomenon. A longer observational baseline is needed to investigate possible periodicity and the frequency of the phenomenon.

Observed period jitter in the Kepler Cepheid V1154 Cygni (Derekas et al. 2012) and flickering observed in two Cepheids with MOST (Evans et al. 2014) suggests that RV modulations have a photometric counterpart. However, no Cepheid has as of yet been found to exhibit both RV modulation and photometric flickering. Only the long-period Cepheid RS Puppis exhibits both strong stochastic period variations and RV modulation. Both phenomena are obvious in the unprecedented Coralie RV data set.

In conclusion, significant improvements in BW distance accuracy may be achieved by determining linear and angular radius variations contemporaneously, if RV curve modulations directly correlate with modulations in angular diameter. Dense contemporaneous time-series of high-quality spectroscopic, photometric, and interferometric measurements are required to investigate this exciting possibility.

\section{References}

Anderson, R. I. 2014, A\&\&A 566, L10

Derekas, A., Szabó, G. M., Berdnikov, L., et al. 2012, MNRAS 425, 1312

Evans, N. R., Szabó, R., Szabados, L., et al. 2014, in J. A. Guzik, W. J. Chaplin, G. Handler, \& A. Pigulski (eds.), IAU Symposium, Vol. 301 of IAU Symposium, pp 55-58

Gieren, W., Storm, J., Nardetto, N., et al. 2013, in R. de Grijs (ed.), IAU Symposium, Vol. 289 of IAU Symposium, pp 138-144

Leavitt, H. S. \& Pickering, E. C. 1912, Harvard College Observatory Circular 173, 1

Nardetto, N., Gieren, W., Kervella, P., et al. 2009, A\&SA 502, 951

Riess, A. G., Macri, L., Casertano, S., et al. 2011, ApJ 730, 119 